\title{
Human Intestinal Lipoproteins
}

\author{
STUDIES IN CHYLURIC SUBJECTS
}

\author{
Peter H. R. Green, Robert M. Glickman, Christopher D. Saudek, \\ Conrad B. Blum, and Alan R. Tall, Gastrointestinal Unit, College of \\ Physicians \& Surgeons of Columbia University, New York 10032; Department of \\ Medicine, Cornell University Medical College, New York 10021
}

A B S T RACT To explore the role of the human intestine as a source of apolipoproteins, we have studied intestinal lipoproteins and apoprotein secretion in two subjects with chyluria (mesenteric lymphatic-urinary fistulae). After oral corn oil, apolipoprotein A-I (apoA-I) and apolipoprotein A-II (apoA-II) output in urine increased in parallel to urinary triglyceride. One subject, on two occasions, after $40 \mathrm{~g}$ of corn oil, excreted 8.4 and $8.6 \mathrm{~g}$ of triglyceride together with 196 and $199 \mathrm{mg}$ apoA-I and on one occasion, $56 \mathrm{mg}$ apoA-II. The other subject, after $40 \mathrm{~g}$ corn oil, excreted $0.3 \mathrm{~g}$ triglyceride and 17.5 $\mathrm{mg}$ apoA-I, and, after $100 \mathrm{~g}$ of corn oil, excreted $44.8 \mathrm{mg}$ apoA-I and $5.8 \mathrm{mg}$ apoA-II. $14.5 \pm 2.1 \%$ of apoA-I and $17.7 \pm 4.3 \%$ of apoA-II in chylous urine was in the $d$ $<1.006$ fraction (chylomicrons and very low density lipoprotein). Calculations based on the amount of apoA-I and apoA-II excreted on triglyceride-rich lipoproteins revealed that for these lipid loads, intestinal secretion could account for 50 and $33 \%$ of the calculated daily synthetic rate of apoA-I and apoA-II, respectively. Similarly, subject 2 excreted $48-70 \%$ and $14 \%$ of the calculated daily synthetic rate of apoA-I and apoA-II, respectively.

Chylous urine contained chylomicrons, very low density lipoproteins and high density lipoproteins, all of which contained apoA-I. Chylomicrons and very low density lipoproteins contained a previously unreported human apoprotein of $46,000 \mathrm{~mol} w \mathrm{w}$. We have called this apoprotein apoA-IV because of the similarity of its molecular weight and amino acid composition to rat apoA-IV. In sodium dodecyl sulfate gels, chylomicron apoproteins consisted of apoB $3.4 \pm 0.7 \%$, apoA-IV 10.0 $\pm 3.3 \%$, apoE $4.4 \pm 0.3 \%$, apoA-I $15.0 \pm 1.8 \%$, and apoC and apoA-II $43.3 \pm 11.3 \%$. Very low density lipoprotein contained more apoB and apoA-IV and less apoC than chylomicrons. Ouchterlony immunodiffusion of chylo-

Received for publication 13 October 1978 and in revised form 1 March 1979. micron apoproteins revealed the presence of apoC-I, apoC-II, and apoC-III. In contrast, plasma chylomicrons isolated during a nonchyluric phase revealed a markedly altered chylomicron apoprotein pattern when compared with urinary chylomicrons. The major apoproteins in plasma chylomicrons were apoB, apoE, and the $\mathrm{C}$ peptides: no apoA-I or apoA-IV were present in sodium dodecyl sulfate gels indicating that major changes in chylomicron apoproteins occur during chylomicron metabolism. When incubated in vitro with plasma, urinary chylomicrons lost apoA-I and apoA-IV and gained apoE and apoC. Loss of apoA-I and apoA-IV was dependent upon the concentration of high density lipoproteins in the incubation mixture.

These studies demonstrate that the human intestine secretes significant amounts of apoA-I and apoA-II during lipid absorption. Subsequent transfer of apoproteins from triglyceride-rich lipoproteins to other plasma lipoproteins may represent a mechanism whereby the intestine contributes to plasma apoprotein levels.

\section{INTRODUCTION}

Animal studies have demonstrated that the small intestine is a site of synthesis of chylomicrons (1), very low density lipoproteins (VLDL) $)^{1}(2,3)$ and high density lipoproteins (HDL) $(4,5)$. During chylomicron formation, rat intestine actively synthesizes three chylomicron apolipoproteins: apoB, apoA-I, and apoA-IV (4, 6-9). The lower molecular weight C peptides and the arginine-rich peptide (apoE) do not appear to be actively synthesized and are transferred to the chylomicron surface after secretion. ApoA-I, the principle apoprotein of plasma HDL, is the major apoprotein

\footnotetext{
${ }^{1}$ Abbreviations used in this paper: ApoA-I, A-II, etc., apolipoprotein A-I, A-II, etc.; apoE, the arginine-rich peptide; DTNB, dithionitrobenzoic acid; HDL, high density lipoprotein; LCAT, lecithin:cholesterol acyltransferase; SDS, sodium dodecyl sulfate; VLDL, very low density lipoprotein.
} 
of rat mesenteric lymph chylomicrons, VLDL and HDL (4-7), and markedly increases in mesenteric lymph during triglyceride absorption (7). In the rat, the quantitative measurement of apoA-I in mesenteric lymph indicates that the intestine is a major synthetic site for apoA-I. In comparison, rat liver appears to have a more limited capacity to synthesize apoA-I (10).

The role of the human intestine in lipoprotein and apoprotein synthesis is less clear, in large measure because of the difficulty in obtaining nascent intestinal lipoproteins in man. Chylomicrons isolated from chylous effusions $(11,12)$ and human thoracic duct lymph (13) have been shown to contain all major chylomicron apoproteins (apoB, apoA-I, apoA-II, apoE, apoC); however, it was unclear which of these apoproteins were synthesized by the intestine or transferred to the chylomicron surface after secretion. In addition, thoracic duct lymph contains hepatic lymph, making the intestinal origin of a given apoprotein difficult to prove. There are few data directly demonstrating human intestinal apoprotein synthesis. A recent study has shown radioactive amino acid incorporation in vitro by human small bowel biopsies into apoB, aopA-I, and apoA-II (14). Immunofluorescence studies with isolated human intestinal epithelial cells have demonstrated immunofluorescence for apoA-I (15) and apoB (16) which increases markedly during lipid absorption, consistent with active synthesis of these apoproteins. In addition, lipid absorption and chylomicronemia results in a transient but significant increase in plasma apoA-I levels (15) suggesting an intestinal contribution to systemic apoprotein levels.

Thus, although human small intestine can synthesize several chylomicron apoproteins, the quantitative importance of this is unknown. We have therefore, studied human intestinal lipoprotein secretion in two chyluric subjects. Lipid absorption has been studied previously in chyluric subjects $(17,18)$. The chyluria in our subjects was the result of fistulous connections between mesenteric lymphatic channels and the renal collecting system and, in both subjects, was considered to be secondary to previous filarial infestation. The lipoproteins isolated from the urine of these subjects had little opportunity to undergo apoprotein exchange with serum lipoproteins and therefore may be more representative of mesenteric lymph lipoproteins.

In particular, we have quantitated the secretion of two major chylomicron apoproteins, apoA-I and apoA-II, after lipid feeding. Our results indicate that human intestine is a major synthetic source for these apoproteins. In addition, a previously undescribed apoprotein, analogous to apoA-IV in the rat, is described in triglyceride-rich (chylomicrons and VLDL) lipoproteins of intestinal origin.

\section{METHODS}

\section{Subjects and collections of samples}

Subject 1. L.C. is a 27-yr-old Chinese female with an 8-yr history of chyluria considered to be the result of previous filarial infestation. Her chief complaint has been weight loss (current weight $41 \mathrm{~kg}$ ) because of persistent chyluria. There was no peripheral edema. Serum albumin was $2.8 \mathrm{~g} / \mathrm{dl}$, globulins $1.9 \mathrm{~g} / \mathrm{dl}$, cholesterol $190 \mathrm{mg} / \mathrm{dl}$, triglyceride $66 \mathrm{mg} / \mathrm{dl}$, fasting plasma apoA-I $166 \mathrm{mg} / \mathrm{dl}$, and apoA-II $41 \mathrm{mg} / \mathrm{dl}$. Normal range for apoA-I is $149-174 \mathrm{mg} / \mathrm{dl}$ and for apoA-II, 25-39 $\mathrm{mg} / \mathrm{dl}$. Lymphangiography demonstrated connections between mesenteric lymphatics and the left renal calyceal system.

Subject 2. J.B., a 61-yr-old black male from Guyana, had been treated for filariasis $20 \mathrm{yr}$ earlier and had a long history of intermittent chyluria. He was otherwise well and maintained a stable weight $(70 \mathrm{~kg})$. Laboratory investigations showed normal plasma proteins, cholesterol $260 \mathrm{mg} / \mathrm{dl}$, triglycerides 60 $\mathrm{mg} / \mathrm{dl}$, fasting plasma apoA-I $145 \mathrm{mg} / \mathrm{dl}$, and apoA-II $33 \mathrm{mg} / \mathrm{dl}$. Lymphangiography revealed mesenteric connections with the left renal calyceal and collecting dust system.

Each subject gave informed consent for the studies which were approved by the Human Studies Committee of New York Hospital.

Corn oil (40 or $100 \mathrm{~g}$ ) was given to each subject on two occasions. Urine was collected after an overnight fast, at which time the urine was clear. After lipid ingestion, urine was collected at 3-h intervals, sodium azide was added, and the samples were refrigerated immediately. Aliquots of whole urine were stored at $-80^{\circ} \mathrm{C}$ for apoprotein quantitation. Lipoproteins were isolated from freshly collected urine. Urine was collected in the presence of dithionitrobenzoic acid (DTNB) in phosphate-buffered saline, $\mathrm{pH} 7.4$, added to a final concentration of $1 \mathrm{mM}$, to inhibit the action of the enzyme lecithin:cholesterol acyltransferase (LCAT) (19). LCAT activity was not measured in these studies.

J.B. had intermittent chyluria during the study. During a nonchyluric phase, he was given oral corn oil (100 g), and plasma was drawn at 2-h intervals for triglyceride and apoA-I quantitation. At $6 \mathrm{~h}$ after corn oil ingestion, a chylomicron fraction was prepared from plasma.

\section{Isolation of lipoproteins}

Lipoproteins were isolated in the Beckman preparative ultracentrifuge model L5-75 (Beckman Instruments, Inc., Spinco Div., Palo Alto, Calif.) with the SW 27 and $50.2 \mathrm{Ti}$ rotors. Chylomicrons were isolated by a single ultracentrifugation at $3 \times 10^{6} \mathrm{~g} \cdot \mathrm{min}$, in the SW 27 rotor, separated from the infranate by means of a tube slicer and purified by passage through $2 \%$ agarose columns packed with Bio-gel A-50m ( 100 200 mesh) obtained from Bio-Rad Laboratories, Richmond, Calif. The columns were eluted with $0.15 \mathrm{M} \mathrm{NaCl}$. VLDL $(d<1.006)$ were isolated by ultracentrifugation at $1 \times 10^{8} \mathrm{~g}$. min and purified either by recentrifugation or by passage through $2 \%$ agarose columns. HDL was isolated between $d$ 1.063 and $1.21 \mathrm{~g} / \mathrm{ml}$ and purified by recentrifugation at the upper density limit. HDL was then dialyzed against $0.15 \mathrm{M}$ $\mathrm{NaCl}$ containing $0.01 \mathrm{M}$ EDTA and sodium azide.

Plasma chylomicrons were isolated from the plasma of J.B. by ultracentrifugation $\left(3 \times 10^{6} \mathrm{~g} \cdot \mathrm{min}\right)$ in the $S W 27$ rotor and purified by agarose column chromatography.

To determine the distribution of apoA-I and apoA-II in whole chylous urine, samples were subjected to sequential ultracentrifugation in the SW 27 rotor to remove chylomicrons 
$\left(3 \times 10^{6} \mathrm{~g} \cdot \mathrm{min}\right)$ and VLDL $\left(1 \times 10^{8} \mathrm{~g} \cdot \mathrm{min}\right)$. Quantitative immunoelectrophoresis was performed on the unwashed chylomicron and VLDL fractions and the $d>1.006$ fraction.

\section{Polyacrylamide gel electrophoresis}

Isolated lipoproteins were delipidated in ethanol:ether $(3: 2$, $\mathrm{vol} / \mathrm{vol}$ ) at $4^{\circ} \mathrm{C}$, the apoproteins subjected to sodium dodecyl sulfate (SDS) polyacrylamide gel electrophoresis ( $5.6 \%$ gels) and stained with Coomassie Blue (1). Chylomicron apoproteins were incubated in the presence of dithiothreitol in a final concentration of $40 \mathrm{mM}$ before gel electrophoresis. Apoproteins were quantified densitometrically as described (1) and were identified by their electrophoretic mobility as compared with purified apoproteins subjected to simultaneous gel electrophoresis. Isoelectric focusing was performed in $7.5 \%$ polyacrylamide gels containing $8 \mathrm{M}$ urea and $0.6 \% \mathrm{pH}$ $3.5-5.0$ ampholyte, $0.6 \%$ pH 5-7 ampholyte, $0.8 \%$ pH 3.5-10 ampholyte at $4^{\circ} \mathrm{C}$ overnight. The gels were stained in $0.04 \%$ Coomassie Blue $\mathrm{G} 250$ in $3.4 \%$ perchloric acid.

\section{Immunological studies}

ApoA-I was quantitated in whole urine or subfractions with the quantitative immunoelectrophoretic technique of Laurell (20). Samples were delipidated with 1,1,3,3-tetramethylurea. The assay was performed as described (15) with the technique described by Albers et al. (21). The assay was linear with dilutions of whole plasma, HDL, and purified apoA-I. A standard of known apoA-I content was used on each plate. ApoA-II was measured by radial immunodiffusion by the technique of Albers et al. $(21,22)$.

Apoproteins were identified by Ouchterlony analysis with monospecific antisera to apoA-I, apoA-II, apoC-I, apoC-II, and apoC-III.

\section{Lipid analysis}

Lipids were extracted in chloroform/methanol $(2: 1, \mathrm{vol} / \mathrm{vol})$ according to the method of Folch et al. (23). Lipid composition was determined by quantitative thin-layer chromatography by the method of Downing (24) as modified by Katz et al. (25). A photodensitometer model $52 \mathrm{C}$ (Photovolt Corp., New York) was used. Cholesterol, cholesterol ester, triacylglycerol, and fatty acids were separated in the solvent system of hexane, diethyl ether, and acetic acid (70:30:1 vol/vol). Individual phospholipids were developed in chloroform, methanol, water, and acetic acid (65:25:4:1 vol/vol). Protein determinations were performed according to the method of Lowry et al. (26). Triglyceride was determined by the AutoAnalyzer I (Technicon Instruments Corporation, Tarrytown, N. Y.) technique (27).

\section{Incubation of chylomicrons with serum and serum lipoproteins}

Purified urinary chylomicrons $(0.2-0.58 \mathrm{mg}$ chylomicron protein) were mixed in conical glass tubes with fresh whole serum $(6-10 \mathrm{ml})$, various lipoprotein fractions, or $0.15 \mathrm{M}$ saline. Incubation was usually performed at $37^{\circ} \mathrm{C}$ by gentle agitation for 20-30 min in a water bath to maintain dispersion of particles. The incubation was stopped by placing the tubes in ice. Some incubations were carried out at $4^{\circ} \mathrm{C}$ and others for shorter periods ( 2 and $10 \mathrm{~min}$ ). Chylomicrons were separated immediately by ultracentrifugation and purified by col- umn chromatography with $2 \%$ agarose columns. In each experiment, chylomicrons incubated in a comparable volume of saline and purified by the same method served as the control.

To explore the role of HDL in the exchange of chylomicron apoproteins, chylomicrons $(0.35 \mathrm{mg})$ were incubated with the $d>1.21 \mathrm{~g} / \mathrm{ml}$ fraction of serum with added HDL in varying concentrations $(0.05-1.5 \mathrm{mg} / \mathrm{ml})$. Some experiments were conducted in the presence of an LCAT inhibitor, DTNB. Incubations were performed at $37^{\circ} \mathrm{C}$ for $30 \mathrm{~min}$, and chylomicrons, as well as HDL, isolated from the incubation mixture were purified as described above, and the apoproteins examined by polyacrylamide gel electrophoresis.

\section{Isolation and characterization of ApoA-IV}

Polyacrylamide-gel electrophoresis of chylomicrons isolated from the chylous urine revealed an apoprotein of 46,000 mol wt. This protein was isolated by preparative electrophoresis (Polyprep 100, Buchler Instruments Div., Searle Diagnostics, Fort Lee, N. J.) by using $5.6 \%$ polyacrylamide gel in SDS. Delipidated chylomicron apoproteins were solubilized in $0.2 \mathrm{M}$ tris buffer, $\mathrm{pH} 7.2$, containing $0.1 \mathrm{M}$ decyl sulfate and at times incubated in the presence of dithiothreitol before application to the preparative gel.

The purified apoprotein was tested by Ouchterlony analysis against antisera to other known apoproteins (apoA-I, apoA-II, apoC-I, apoC-II, apoC-III, apoE). Amino acid analysis was kindly performed by Dr. Steinman, Albert Einstein College of Medicine, N. Y., with a Beckman 120B Amino Acid Analyzer (Beckman Instruments).

\section{RESULTS}

\section{ApoA-I and apoA-II secretion}

Each subject received a test meal of corn oil after an overnight fast. Urine was collected at 3-h intervals. As shown in Fig. 1, lipid ingestion was associated with a subsequent rise in urinary triglyceride in both subjects. Subject 1 (L.C.) showed a peak urinary triglyceride of $2.7 \mathrm{~g} / \mathrm{h}$ by $6 \mathrm{~h}$, whereas the triglyceride content of the urine of subject 2 (J.B.) showed a more modest rise. Also shown (Fig. 1) are the hourly secretion of apoA-I and apoA-II. In both subjects there was a rise in the content of both apoproteins with lipid feeding which paralleled urinary triglyceride.

Table I shows the quantitative output of triglyceride and apoproteins in both subjects on two separate occasions. Patient L.C. excreted 8.4 and $8.6 \mathrm{~g}$ of triglyceride after $40 \mathrm{~g}$ of corn oil on two occasions, representing $\cong 20 \%$ of the ingested fat. Total apoA-I appearing in the urine on each occasion was similar at 196-199 mg. Total urinary apoA-II was $29 \mathrm{mg}$ during one test period. The mean mass ratio of apoA-I:apoA-II in whole urine was $7.1 \pm 0.1( \pm \mathrm{SEM}, n=6)$ as determined from six collection periods.

Chyluria in patient J.B. was less marked and intermittent. $40 \mathrm{~g}$ oral corn oil resulted in total urinary triglyceride output of $305 \mathrm{mg}$, whereas apoA-I output was $17.5 \mathrm{mg}$. On a second occasion, $100 \mathrm{~g}$ corn oil resulted 


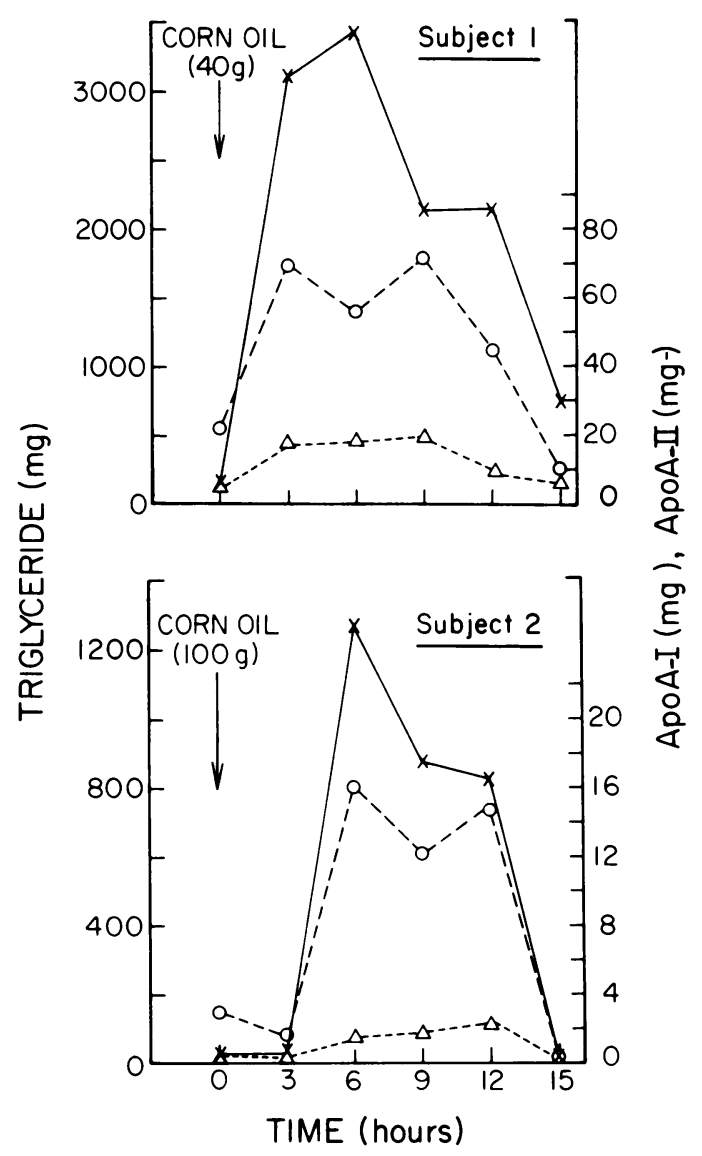

Figure 1 Human chylous urine output of triglyceride, apoA-I, and apoA-II after corn oil feeding. Each subject received corn oil after an overnight fast, and urine was collected at 3-h intervals. Triglyceride $(\times)$, apoA-I $(O)$, and apoAII $(\triangle)$ were measured (see Methods). ApoA-II is plotted as milligrams $\times 2$.

in a total output of $2.9 \mathrm{~g}$ triglyceride and $44.8 \mathrm{mg}$ apoA-I over a 12 -h period. During this period $3.2 \mathrm{mg}$ of apoA-II was excreted. The mean mass ratio of apoA-I and apoA-II in whole urine was $14.2 \pm 1.0( \pm \operatorname{SEM}, n=4)$. When studied during a nonchyluric phase, oral corn oil (100 g) resulted in a rise in plasma triglyceride from 68 to 121 $\mathrm{mg} / \mathrm{dl}$, whereas plasma apoA-I rose from 145 to 159 $\mathrm{mg} / \mathrm{dl}$ at $6 \mathrm{~h}$.

To determine whether normal urine contains apoA-I, samples of urine from four normal volunteers were concentrated 3-15 times and subjected to quantitative immunoelectrophoresis. No apoA-I was detected in these concentrated samples. Normal plasma diluted with urine had exactly the same rocket height as plasma diluted with saline or $0.01 \mathrm{M}$ tris buffer in $8 \mathrm{M}$ urea, indicating that urine itself did not affect the assay for apoA-I.
TABLE I

Output of Triglyceride and Apoproteins in Chylous Urine

\begin{tabular}{ccccc}
\hline Subject & $\begin{array}{c}\text { Collection } \\
\text { period }\end{array}$ & $\begin{array}{c}\text { Urine } \\
\text { triglyceride }\end{array}$ & apoA-I & apoA-II \\
\hline $\begin{array}{c}\text { Subject } 1 \\
\text { (L.C.) }\end{array}$ & $h$ & $g$ & $m g$ & $m g$ \\
& $9^{*}$ & 8.4 & 196 & Not measured \\
$\begin{array}{c}\text { Subject } 2 \\
\text { (J.B.) }\end{array}$ & $12^{*}$ & 8.6 & 199 & 29 \\
& $9^{*}$ & 0.3 & 17.5 & Not measured \\
& $12 \ddagger$ & 2.9 & 44.8 & 3.2 \\
\hline
\end{tabular}

Each patient received oral corn oil, and urine was collected in 3-h time periods. Triglyceride, apoA-I, and apoA-II were quantitated as described in Methods.

$* 40 \mathrm{~g}$ oral corn oil given.

$\$ 100 \mathrm{~g}$ oral corn oil given.

\section{Distribution of apoA-I and apoA-II in chylous urine}

To determine the density distribution of apoproteins in chylous urine, chylomicrons and VLDL were removed from chylous urine of both patients by sequential ultracentrifugation as described in Methods. Rocket immunoelectrophoresis was performed on the unwashed fractions. $14.5 \pm 2.1 \%$ of apoA-I was in the $d$ $<1.006$ fraction (chylomicrons and VLDL), whereas $86 \pm 3.3 \%(n=6)$ was present in the $d>1.006$ fraction. ApoA-II was similarly distributed with $17.7 \pm 4.3 \%$ present in the $d<1.006$ fraction and $81.9 \pm 4 \%$ in the $d$ $>1.006$ fraction $(n=5)$.

\section{Characterization of lipoproteins from chylous urine}

Chylomicrons, VLDL, and HDL were isolated from the urine of both patients by ultracentrifugation, and the apoproteins characterized by SDS polyacrylamide gel electrophoresis (Figs. 2 and 3).

Chylomicrons. The urinary chylomicrons were isolated by a single ultracentrifugal step and purified by agarose column chromatography. Both subjects had similar chylomicron apoprotein patterns (Figs. 2 and 3). The major apoproteins as judged by their mobility in SDS gels were apoB, apoA-I, an apoprotein of 46,000 mol wt (apoA-IV characterized below), and the C peptides. ApoE was a minor apoprotein. The distribution of apoproteins as determined by densitometric scanning of stained gels is shown in Table II. The prior incubation of apoproteins with dithiothreitol did not alter the percent apoprotein composition. This gel system does not adequately separate apoA-II from the C peptides; however, Ouchterlony immunodiffusion against monospecific antisera revealed the presence of 


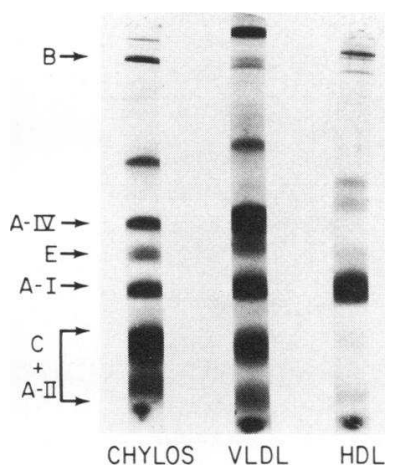

FIGURE 2 SDS polyacrylamide gels of urinary lipoproteins from subject 1 (L.C.) obtained after oral corn oil. Chylomicrons and VLDL were isolated at $3 \times 10^{6} \mathrm{~g} \cdot \mathrm{min}$, respectively, and purified by passage through $2 \%$ agarose. HDL (1.063-1.21) was purified by recentrifugation.

apoA-II, apoC-I, apoC-II, and apoC-III. The ratio of apoA-I:apoA-II in chylomicrons determined by rocket immunoelectrophoresis was 5.2 for L.D. and 13.1 for J.B. To compare the apoprotein pattern of chylomicrons isolated from the serum and urine of the same subject, chylomicrons were isolated from the serum of J.B. when chyluria had ceased and, in marked contrast to the urinary chylomicrons, the major apoproteins of serum chylomicrons were apoB, apoE, and the C peptides (Fig. 3). ApoA-I and apoA-IV were not detected in these plasma chylomicrons, suggesting a loss of these apoproteins from the chylomicron surface upon entry to plasma.

The lipid composition of chylomicrons is shown in Table III. The total cholesterol, $1.6 \%$, consisted of

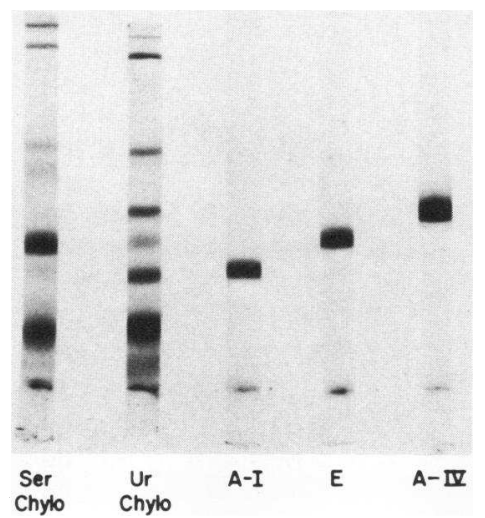

FIGURE 3 SDS polyacrylamide gels of serum chylomicrons (Ser Chylo) and urinary chylomicrons (Ur Chylo) from subject 2 (J.B.) obtained after oral corn oil. ApoA-I, apoE, and apoAIV were isolated by SDS preparative electrophoresis of delipidated urinary chylomicron apoproteins. SDS gels were run after incubation of apoproteins with dithiothreitol in a final concentration of $40 \mathrm{mM}$, for $1 \mathrm{~h}$.
TABLE II

Apoprotein Composition of Chylomicrons and VLDL (Chylous Urine)

\begin{tabular}{lccc}
\hline & $\begin{array}{c}\text { Chylomicrons } \\
(n=7)\end{array}$ & $\begin{array}{c}\text { VLDL } \\
(n=5)\end{array}$ & $\begin{array}{c}\text { Chylomicrons } \\
\text { incubated } \\
\text { with serum } \\
(n=4)\end{array}$ \\
\hline apoB & $3.4 \pm 0.7$ & $10.9 \pm 1.9^{*}$ & $3.6 \pm 0.8$ \\
apoA-IV & $10.0 \pm 3.3$ & $24.6 \pm 2.8^{*}$ & $1.6 \pm 0.6^{*}$ \\
apoE & $4.4 \pm 0.3$ & $6.0 \pm 0.3$ & $14 \pm 1.7^{*}$ \\
apoA-I & $15.0 \pm 1.8$ & $16.5 \pm 2.0$ & $2.8 \pm 0.3^{*}$ \\
apoC and apoA-II & $47.3 \pm 11.3$ & $30.3 \pm 3.5^{*}$ & $57.3 \pm 9.1$ \\
\hline
\end{tabular}

Chylomicrons and VLDL from the chylous urine of both patients were delipidated and subjected to polyacrylamide gel electrophoresis in the presence of dithiothreitol. Apoproteins were quantitated densitometrically. Values represent the percentage distribution of area under the absorbance tracing. Conditions of chylomicron incubation experiments are described in the text.

${ }^{*} P<0.05$ Students $t$ test compared to chylomicrons.

equal amounts of free and esterified cholesterol. Phospholipids comprised $6.3 \%$, triglyceride $91.5 \%$, and protein $1.33 \%$ of chylomicron mass. This lipid composition is similar to that reported by others for human chylomicrons $(12,13)$.

$V L D L$. Lipoproteins isolated at $1 \times 10^{8} \mathrm{~g} \cdot \mathrm{min}$ after the removal of chylomicrons $\left(\mathrm{S}_{\mathrm{f}}>400\right)$ are designated $\operatorname{VLDL}\left(\mathrm{S}_{\mathrm{f}}=20-400\right)$. VLDL were present in the urine of L.C. while fasting and in the urine of both L.C. and J.B. when chyluric. The apoprotein pattern of VLDL resembled chylomicrons (Fig. 2), and the apoprotein composition assessed densitometrically is shown in Table II. VLDL from fasting urine and chylous urine had a similar apoprotein composition. There was comparatively more apoB and apoA-IV and proportionally less low molecular weight apoproteins than in chylomicrons. The lipid composition of VLDL presumably of intestinal origin, revealed triglyceride $73.3 \%$, phospholipids $15.5 \%$, free cholesterol $2.3 \%$, and cholesterol ester $5.8 \%$. Protein constituted $4 \%$ of the mass. The increase in polar constituents is consistent with the smaller size of VLDL. The cholesterol content of VLDL was greater than chylomicrons. The free cholesterol:cholesterol ester ratio of VLDL was 0.4 compared with a chylomicron free cholesterol:cholesterol ester ratio of 1 . Similar free cholesterol:cholesterol ester ratios were observed by Ockner et al. (3) for rat mesenteric lymph chylomicrons and VLDL.

HDL. HDL were isolated between the $d 1.063$ and $1.21 \mathrm{~g} / \mathrm{ml}$ from the urine obtained while fasting and after corn oil. The major apoprotein of HDL was apoA-I and the apoprotein pattern in SDS gels resembled plasma HDL. However, the ratio of apoA-I:apoA-II in 
TABLE III

Lipid Composition of Lipoproteins from Chylous Urine*

\begin{tabular}{|c|c|c|c|c|c|c|c|}
\hline & $\begin{array}{c}\begin{array}{c}\text { Free } \\
\text { cholesterol }\end{array}\end{array}$ & $\begin{array}{l}\text { Fatty } \\
\text { acids }\end{array}$ & Triglyceride & $\begin{array}{l}\text { Cholesterol } \\
\text { ester }\end{array}$ & Sphingomyelin & $\begin{array}{l}\text { Phosphatidyl- } \\
\text { choline }\end{array}$ & Proteint \\
\hline & \multicolumn{6}{|c|}{$\%$ of total lipid } & $\%$ \\
\hline Chylomicrons $(n=6)$ & $0.8 \pm 0.2$ & $0.2 \pm 0.1$ & $91.5 \pm 1.1$ & $0.8 \pm 0.2$ & - & $6.3 \pm 1.3$ & 1.33 \\
\hline $\operatorname{VLDL}(n=4)$ & $2.3 \pm 0.5$ & $2.2 \pm 0.7$ & $73.3 \pm 4.3$ & $5.8 \pm 2.0$ & $0.8 \pm 0.4$ & $14.7 \pm 3.5$ & 4.04 \\
\hline $\begin{array}{l}\text { Urinary HDL with added DTNB } \\
(n=6)\end{array}$ & $8.2 \pm 0.9$ & & $11.1 \pm 2.0$ & $38.0 \pm 3.6$ & $6.9 \pm 1.4$ & $34.6 \pm 3.6$ & 53.5 \\
\hline Serum HDL $(n=10)$ & $5.12 \pm 1.16$ & - & $2.8 \pm 0.83$ & $37.0 \pm 3.83$ & $6.91 \pm 1.09$ & $46.2 \pm 3.23$ & 51.3 \\
\hline
\end{tabular}

Lipoproteins from each subject were used.

* All values are mean \pm SEM. Percentage composition estimated by quantitative thin-layer chromatography.

$\$$ Protein:lipid + protein $\times 100 \%$.

HDL from chylous urine was 5.6 for L.C. and 7.1 for J.B. No apoA-IV was present in SDS gels. The lipid analysis of HDL isolated from urine collected in the presence of DTNB (Table III) was identical to that collected in the absence of DTNB, and resembled plasma HDL. Negative stain electron microscopy was performed on several urinary HDL samples. No discoid HDL particles were seen, which is consistent with the lipid composition of the fraction; however, the addition of DTNB to voided urine would not have prevented the activity of LCAT if present within the bladder.

\section{Characterization of ApoA-IV}

As noted above (Figs. 2 and 3, Table II) the apoprotein pattern of delipidated $d<1.006$ lipoproteins obtained from chyluric urine revealed the presence of an apoprotein of $46,000 \mathrm{~mol}$ wt which accounted for 10 and $24 \%$ of chylomicron and VLDL protein, respectively. This apoprotein was purified by preparative SDS acrylamide electrophoresis (Fig. 3). Treatment of the apoprotein with the reducing agents dithiothreitol or $\beta$-mercaptoethanol either before or after purification did not alter its molecular weight or result in the appearance of additional protein bands in SDS gels. There was no reaction against antisera to apoA-I, apoA-II, apoE, apoC-I, apoC-II, or apoC-III when tested by Ouchterlony immunodiffusion. These studies indicate that this apoprotein is not a polymer of lower molecular weight apoproteins. Amino acid analysis of the purified apoprotein is shown in Table IV. The values obtained are compared with published values for rat apoA-IV obtained from chylomicrons (28) and plasma HDL (29) and are similar except for some minor discrepancies such as an increased number of tyrosine residues. We have therefore designated this apoprotein, apoA-IV. Isoelectric focusing of purified apoA-IV revealed the presence of three bands, one major band with a $\mathrm{pI}$ $=5.65$ and two minor bands at $\mathrm{pH} 5.73$ and 5.57. SDS gels of normal plasma VLDL and HDL did not show the presence of this apoprotein. Preliminary quantitative studies reveal that apoA-IV was not present in concentrated normal urine. In addition, we have noted in normal volunteers a postprandial rise in total serum apoA-IV levels (unpublished observations); however, plasma chylomicrons isolated from the lipemic plasma of one chyluric subject (J.B.) did not contain ApoA-IV (Fig. 3) suggesting a loss of this apoprotein from the chylomicron surface after secretion. To explore the transfer of chylomicron apoproteins in more detail, a series of in vitro incubation studies were carried out.

TABLE IV

Amino Acid Composition of Human Chylomicron ApoA-IV

\begin{tabular}{lccr}
\hline \multicolumn{1}{c}{ Amino acid } & $\begin{array}{c}\text { Human chylomicron } \\
\text { apoA-IV }\end{array}$ & $\begin{array}{c}\text { Rat chylomicron } \\
\text { apoA-IV* }\end{array}$ & $\begin{array}{r}\text { Rat HDL } \\
\text { apoA-IVI }\end{array}$ \\
\hline & & & \\
Lysine & & & \\
Histidine & 80 & 73 & 67 \\
Arginine & 14 & 15 & 16 \\
Aspartic acid & 40 & 37 & 40 \\
Threonine & 93 & 133 & 123 \\
Serine & 58 & 53 & 52 \\
Glutamic acid & 92 & 56 & 54 \\
Proline & 174 & 216 & 203 \\
Half-cystine & 38 & 44 & 41 \\
Glycine & ND & ND & 5 \\
Alanine & 45 & 42 & 48 \\
Valine & 94 & 63 & 65 \\
Methionine & 62 & 60 & 64 \\
Isoleucine & 19 & 20 & 23 \\
Leucine & 16 & 18 & 24 \\
Tyrosine & 100 & 126 & 122 \\
Phenylalanine & 29 & 11 & 14 \\
Tryptophan & 44 & 34 & 33 \\
\hline & & & \\
\hline
\end{tabular}

* Imaizumi et al. (28).

$\$$ Swaney et al. (29).

$\S$ Not determined. 
TABLE V

Chylomicron Incubation Protein:Triglyceride Ratios

\begin{tabular}{llll}
\hline \multicolumn{1}{c}{ Incubation } & $n$ & $\begin{array}{l}\text { Protein:tri- } \\
\text { glyceride* }\end{array}$ & \multicolumn{1}{c}{$P \mathfrak{l}$} \\
\hline Chylomicrons (no incubation) & 5 & $1.01 \pm 0.2$ & \\
Chylomicrons + whole serum & 8 & $2.94 \pm 0.5$ & $<0.0025$ \\
Chylomicrons + 0.15 M saline & 4 & $0.98 \pm 0.1$ & $>0.2$ \\
\hline
\end{tabular}

Chylomicrons $(0.2-0.58 \mathrm{mg})$ were incubated with saline or serum (Methods) and isolated by centrifugation, then purified by passage through $2 \%$ agarose columns.

$*$ wt/wt $\times 100$, mean \pm SEM.

$\ddagger P$ value by students $t$ test for paired values when compared with unincubated chylomicrons.

\section{In vitro chylomicron incubation studies}

The effect of exposure of mesenteric chylomicrons to plasma and plasma lipoprotein fractions was studied. Chylomicrons (0.2-0.58 mg chylomicron protein) were incubated with plasma or saline. The final triglyceride concentrations ranged from 250 to $490 \mathrm{mg} / \mathrm{dl}$. As slight losses of lipids as well as protein occur upon recentrifugation of lipoproteins, total protein is expressed as a fraction of the content of triglycerides. Table $\mathrm{V}$ shows the protein:triglyceride ratio of chylomicrons after incubation with plasma and shows a significant gain in protein (protein:triglyceride $\times 100=2.94 \pm 0.5$ ) when compared with unincubated chylomicrons (protein:triglyceride $\times 100=1.01 \pm 0.1$ ). Chylomicrons incubated with saline and reisolated by the same technique showed no significant change in the protein:triglyceride values. When incubated with $10-20 \mathrm{ml}$ of $d>1.21$ fraction of serum, chylomicrons gained less protein than in the presence of whole plasma (protein:triglyceride ratio $1.40 \pm 0.6, n=3$ ).

As shown in Fig. 4, the apoprotein composition of chylomicrons after exposure to plasma was markedly altered when compared with fresh mesenteric chylomicrons. There was a marked loss of apoA-I and apoA-IV accompanied by a gain of apoE and $C$ peptides. The apoprotein composition of chylomicrons incubated with plasma closely resembled that of chylomicrons isolated from the plasma of the same patient during alimentary lipemia (Fig. 3), the major apoproteins being apoB, apoE, and the $\mathrm{C}$ peptides. Apoprotein quantitation in chylomicrons incubated with plasma was determined by planimetry of SDS gels (Table II). ApoE increased from $4.4 \%$ of the chylomicron protein to $13 \%$, whereas apoA-I decreased from 15 to $2.8 \%$ and apoA-IV decreased from 10 to $1.6 \%$. When expressed as alteration in protein mass, relative to triglyceride,$^{2}$ apoE increased from 4.6 to 41.2 , apoC from 44 to 170 , and

\footnotetext{
${ }^{2}$ Calculated from percent apoprotein $\times$ (protein/triglyceride) $\times 100$.
}

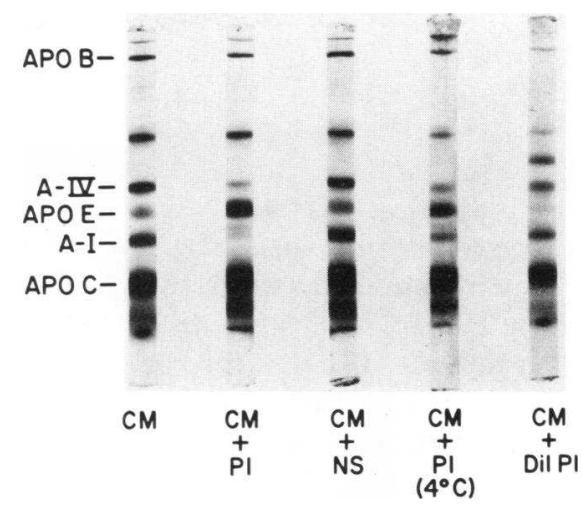

FIGURE 4 SDS polyacrylamide gels of human chylomicrons. Incubation studies. Fresh urinary chylomicrons $(0.2-0.58 \mathrm{mg}$ chylomicron apoprotein) were incubated with plasma (PI), normal saline (NS), plasma at $4^{\circ} \mathrm{C}$, and plasma diluted with saline $(\mathrm{CM}+\mathrm{Dil} \mathrm{Pl})$. Chylomicrons were reisolated $\left(3 \times 10^{6}\right.$ $\mathrm{g} \cdot \mathrm{min})$ and purified by $2 \%$ agarose column chromatography. The apoprotein bands are labeled.

apoA-I decreased from 15.2 to 8.2 reflecting an alteration in total mass of these apoproteins. Thus apoE increased ninefold and $\mathrm{C}$ peptides fourfold.

Also shown in Fig. 4 is the result of chylomicrons incubated with saline and subjected to the same solution and preparative procedure. No change in apoprotein distribution was evident indicating that selective losses of apoproteins did not occur during lipoprotein reisolation. Chylomicrons incubated with plasma at $4^{\circ} \mathrm{C}$ gained apoE and lost apoA-I and apoA-IV suggesting that the apoprotein exchange is not dependent on enzymic action. This process is rapid. When chylomicrons were mixed with serum and reisolated without incubation, apoprotein exchange was noted to have occurred.

To determine which fractions of plasma influenced chylomicron apoprotein transfer, chylomicrons were incubted with various fractions of plasma. Chylomicrons incubated with $d>1.21 \mathrm{~g} / \mathrm{ml}$ fraction of plasma gained apoE; however, there was no obvious loss of apoA-I or apoA-IV under these conditons. When incubated with either $(a)$ whole plasma, $(b)$ the $d>1.006$ fraction of plasma, $(c) \mathrm{HDL}$, or $(d)$ HDL mixed with the $d>1.21$ fraction of plasma, chylomicrons lost apoA-I and apoA-IV. When serum was diluted with saline so that the HDL concentration was equivalent to that in the chylous urine (about $12 \%$ of serum), apoprotein loss did not occur (Fig. 4). It appeared that apoprotein loss from chylomicrons might depend on the concentration of HDL present in the incubation mixture. Experiments were therefore performed in which chylomicrons were incubated with the $d>1.21$ fraction of plasma and varying concentrations of HDL (from 0.05 to $1.5 \mathrm{mg} / \mathrm{ml} \mathrm{HDL}$ protein). The content of chylomicron apoA-I (as detected by quantitative immunoelectrophoresis) decreased with increasing HDL concen- 
tration up to $1.0 \mathrm{mg} / \mathrm{ml}$. At concentrations $>1.0 \mathrm{mg} / \mathrm{ml}$ HDL protein, apoA-I could not be detected in the reisolated chylomicron fraction. In all incubations apoA-IV appeared to parallel apoA-I (Fig. 4). The addition of DTNB to the incubation mixture did not affect apoprotein transfer indicating that the LCAT reaction was not necessary for apoprotein transfer. HDL reisolated from the incubation mixture had a similar apoprotein pattern to the starting HDL. Similar experiments were carried out with mesenteric VLDL $(d<1.006 \mathrm{~g} / \mathrm{ml})$. When incubated with plasma from which VLDL had been removed ( $d>1.006$ fraction), there was a net gain in protein (protein:triglyceride $\times 100$ increased from 4.26 \pm 1.2 to $16.8 \pm 4.5, n=3$ ). VLDL gained apoE and lost apoA-I and apoA-IV. The apoprotein pattern in SDS gels of mesenteric VLDL incubated with $d>1.006$ fraction of plasma resembled the apoprotein pattern of plasma VLDL. Lipoprotein electrophoresis showed that intestinal VLDL consistently migrated closer to the origin than serum VLDL, however, after incubation with $d>1.006$ fraction of serum, intestinal VLDL took on the mobility of serum VLDL (pre- $\beta$ ).

\section{DISCUSSION}

Studies using the mesenteric lymph fistula rat (7) and the isolated perfused liver $(19,30)$ have provided information about the composition of newly secreted lipoproteins before metabolism in the circulation. These studies have also provided some insight into the relative roles of the liver and the intestine in the production of apoA-I, the major apoprotein of plasma HDL. The rat intestine secretes lipoproteins rich in apoA-I $(7,9)$, whereas the isolated perfused liver appears to have a limited capacity to secrete apoA-I (10). In man, chylomicrons isolated from the thoracic duct, peritoneal, and pleural cavities (11-13) contain apoA-I and apoA-II, and it appears that the human intestine synthesizes apoA-I and apoA-II $(14,15)$. However, the quantitative importance of the human intestine as a source of the A proteins is not clear.

The present studies, in two subjects with mesenteric lymphatic-urinary fistulae, have demonstrated that lipid absorption and chylomicron transport in mesenteric lymph are associated with a coincident marked rise in total apoA-I and apoA-II excreted in the urine of these subjects.

The urine of both subjects contained chylomicrons, VLDL, and HDL all of which contained apoA-I and apoA-II. The majority of apoA-I and apoA-II (80-85\%) was present in the nontriglyceride-rich lipoprotein fraction $(d>1.006 \mathrm{~g} / \mathrm{ml})$ of the urine. We cannot be certain of the intestinal origin of this HDL fraction because it resembled plasma HDL both in its lipid and apoprotein composition, and is most likely from multiple sources, including filtered plasma HDL as well as the intestine, although we have no evidence for this in man. The contribution of hepatic lymph lipoproteins to chylous urine cannot be assessed.

It is of interest to calculate the total intestinal secretion of apoA-I and apoA-II in each subject based on the percentage of ingested lipid recovered in the urine. This percentage is considered to represent the precent of mesenteric lymph shunted into the urine (17). We have based these calculations only on the $d<1.006$ lipoproteins recovered in urine because these particles are too large to be filtered from plasma. Additionally, it is assumed that all apoA-I and apoA-II recovered in the $d<1.006$ lipoproteins was derived from the intestine. Animal studies indicate that $<5 \%$ of chylomicron apoA-I is filtered from plasma (31), and our studies indicate that chylomicrons exposed to plasma rapidly lose apoA-I rather than acquire it from plasma HDL. As lipoproteins lose apoproteins upon centrifugation (28), calculations based only on the content of apoA-I and apoA-II in the $d<1.006$ lipoproteins may underestimate the secretion of these apoproteins.

These calculations, based on the $d<1.006$ apoproteins rather than total urinary apoproteins, reveal that subject 1 (L.C.) during the first test period secreted 141 $\mathrm{mg}$ and during the second test period $144 \mathrm{mg}$ of apoA-I over $12 \mathrm{~h}$ after $40 \mathrm{~g}$ of corn oil. This compares with a calculated synthetic rate of apoA-I in this patient of 270 $\mathrm{mg} / \mathrm{d} .^{3}$ Similar calculations for apoA-II indicate that L.C. secreted $26 \mathrm{mg}$ apoA-II during $12 \mathrm{~h}$ which compares with the theoretical apoA-II synthetic rate of $78 \mathrm{mg} / \mathrm{d}^{3}$ Similarly, subject 2 (J.B.) secreted 344 and $244 \mathrm{mg}$ of apoA-I during the first and second test periods, respectively. His calculated synthetic rate of apoA-I is $463 \mathrm{mg} / \mathrm{d} .^{3}$ ApoA-II synthesized during the second test period was $19 \mathrm{mg}$, whereas the theoretical synthetic rate of apoA-II is $133 \mathrm{mg} / \mathrm{d}$. These calculations, although derived with the above reservations, provide an indication that human small intestine is a quantitatively important source of apoA-I and apoAII for plasma. The calculated daily synthetic rates were derived from normal subjects in a steady-state situation (32). Because the subjects with chyluria lose apoA-I and apoA-II into urine, they may have altered synthetic rates for these apoproteins. HDL synthetic rates have not been determined in these subjects. The calculated output of phospholipid from the intestine was 3-6 $\mathrm{g}$, indicating that there is a considerable amount of phospholipid secreted with triglyceride-rich lipoproteins.

As we have previously described for rat mesenteric lipoproteins $(5,7)$, the apoproteins of all lipoproteins prepared from chylous urine (Fig. 2) have apoA-I as a major apoprotein. The apoprotein composition of urinary $d<1.006$ lipoproteins found in the present

\footnotetext{
${ }^{3}$ Calculated assuming an HDL synthetic rate of $8.51 \mathrm{mg} / \mathrm{kg}$ per d and an apoA-I:apoA-II of 3.49:1 for plasma HDL (32).
} 
study agrees with previously published data on thoracic duct chylomicrons $(12,13)$ and confirms that apoB, apoA-I, apoA-II, apoE, and the $\mathrm{C}$ peptides are present. In addition, urinary $d<1.006$ lipoproteins (chylomicrons and VLDL) from the urine of both subjects contained an apoprotein with a molecular weight of 46,000 . We have called this apoprotein apoA-IV because it appears to be analogous to apoA-IV of rat plasma HDL and mesenteric lymph chylomicrons. Rat apoA-IV has a molecular weight of 46,000 and constitutes $10-25 \%$ of plasma HDL apoproteins (29) and about $10 \%$ of mesenteric lymph chylomicron apoprotein (28). It appears to be actively synthesized in the rat intestine as $\left[{ }^{3} \mathrm{H}\right]$ leucine is incorporated into apoA-IV of chylomicrons $(1,9)$ and, there is a marked increase in intracellular fluorescence of apoA-IV during lipid absorption (unpublished observations).

Human apoA-IV has an amino acid composition that resembles that reported for rat apoA-IV isolated from plasma HDL and chylomicrons (Table IV). The amino acid composition does not resemble that reported for other human apoproteins. ApoA-IV migrates as a single band in SDS polyacrylamide gels despite incubation with reducing agents, indicating it is not a polymer of other apoproteins such as apo (E-A-II), a dimer of apoA-II and apoE, which has a similar molecular weight (33). The amino acid composition and nonreducibility of apoA-IV indicates that apoA-IV is not one of the threonine-poor apoproteins of plasma HDL recently reported by Shore et al. (34). ApoA-IV is a major apoprotein of mesenteric $d<1.006$ lipoproteins, however its distribution in plasma and metabolic role remain to be determined. The present studies indicate that apoA-IV as well as apoA-I may leave the chylomicron surface after secretion. As shown in Fig. 3, plasma chylomicrons from the same subject do not contain apoA-I or apoA-IV although urinary chylomicrons do. ApoA-IV is not an artifact of chyluria for we and others (35) have recently noted apoA-IV in chylomicrons and VLDL obtained from human thoracic duct lymph (unpublished observations).

The in vitro incubation studies demonstrate that apoprotein transfer occurs on exposure of human chylomicrons to plasma. Apoprotein transfer occurs at $4^{\circ} \mathrm{C}$ suggesting that it does not involve enzymic action. Transfer involved a gain of apoE (ninefold) and C peptides (fourfold), whereas there was a loss of apoA-I (50\%) and apoA-IV. ApoA-II possibly transfers from the chylomicron surface as well, however this was not addressed in this study. When incubated with the $d$ $>1.21$ fraction of plasma, chylomicrons gained apoE. Whether apoE is normally present in the lipoproteinfree fraction of plasma or is an artifact of centrifugation is not clear. Of importance was the finding that no loss of apoA-I or apoA-IV occurred under these circumstances, suggesting that lipoproteins were in- volved in A protein transfer. Studies with HDL in variable amounts indicate that HDL is the probable acceptor for chylomicron apoA-I. Loss of apoA-I occurs with increasing HDL concentration. Although the HDL concentration in chylous urine is not sufficient for complete apoprotein transfer to occur, some apoA-I may have already left the chylomicron surface. The apoA-I concentration of urinary $d<1.006$ lipoproteins (Table II) may therefore be an underestimate.

The physiological importance of apoprotein transfer observed when chylomicrons are incubated in vitro remains to be determined. However, the apoprotein pattern of chylomicrons isolated from the plasma of one patient closely resembled that of urinary chylomicrons after incubation with plasma. This suggests apoprotein modifications similar to those observed in vitro occur in vivo during chylomicron metabolism. The role of HDL as an acceptor of chylomicron apoA-I is consistent with the intestine being a source of HDL apoA-I. The recent studies of Schaefer et al. (12) which demonstrated transfer of chylomicron apoA-I radioactivity from in vitro labeled human chylomicrons to HDL both during in vitro incubation and in vivo infusion studies are consistent with this concept. Studies in the rat $(36,37)$ show that mass transfer of apoA-I and phospholipid from chylomicrons into HDL occurs during chylomicron catabolism. It therefore appears that mechanisms other than transfer of proteins may also be involved physiologically during chylomicron metabolism.

The present studies provide additional evidence that intestinal lipoproteins in man contribute A apoproteins to plasma lipoproteins during acute lipid feeding. It is likely that under physiological circumstances (100-g fat diet) the intestine contributes major amounts of these apoproteins to plasma. It is also clear that alternative sites of synthesis for these apoproteins exist (i.e., hepatic). The dietary factors that modulate the relative synthetic contribution from each of these sources is undoubtedly complex. For example, high carbohydrate feeding has been shown to increase the catabolism of HDL apoproteins (32), whereas polyunsaturated fat feeding reduced the synthesis of apoA-I (38). Further studies will be required to define the intestinal synthesis of HDL apoproteins under various dietary conditions.

\section{ACKNOWLEDGMENTS}

We thank M. E. Whitlock and E. Quinet for their excellent technical assistance.

This work was supported by National Institutes of Health grants AM 18911, 3P 50HL 21006-0251, Scope E and F, and 2 M01 RR00047.

\section{REFERENCES}

1. Glickman, R. M., and K. Kirsch. 1973. Lymph chylomicron formation during the inhibition of protein synthesis. 
Studies of chylomicron apoproteins. J. Clin. Invest. 52: 2910-2920.

2. Ockner, R. K., F. B. Hughes, and K. J. Isselbacher. 1969. Very low density lipoproteins in intestinal lymph: role in triglyceride and cholesterol transport during fat absorption. J. Clin. Invest. 48: 2367-2373.

3. Ockner, R. K., F. B. Hughes, and K. J. Isselbacher. 1969. Very low density lipoproteins in intestinal lymph: origin, composition and role in lipid transport in the fasting state. J. Clin. Invest. 48: 2079-2088.

4. Windmueller, H. G., and A. E. Spath. 1972. Fat transport and lymph and plasma lipoprotein biosynthesis by isolated intestine. J. Lipid Res. 13: 92-105.

5. Green, P. H. R., A. Tall, and R. M. Glickman. 1978. Rat intestine secretes discoidal HDL. J. Clin. Invest. 61: 528-534.

6. Windmueller, H. G., P. H. Herbert, and R. I. Levy. 1973. Biosynthesis of lymph and plasma lipoprotein apoproteins by isolated perfused rat liver and intestine. J. Lipid Res. 14: 215-223.

7. Glickman, R. M., and P. H. R. Green. 1977. The intestine as a source of apolipoprotein A-I. Proc. Natl. Acad. Sci. U. S. A. 74: 2569-2573.

8. Schonfeld, G., E. Bell, and D. H. Alpers. 1978. Intestinal apoproteins during fat absorption. J. Clin. Invest. 61: 1539- 1550.

9. Wu, A., and H. G. Windmueller. 1978. Identification of circulating apolipoproteins synthesized by rat small intestine in vivo. J. Biol. Chem. 253: 2525-2528.

10. Felker, T. E., M. Fainaru, R. L. Hamilton, and R. J. Havel. 1977. Secretion of the arginine-rich and A-I apolipoproteins by the isolated perfused rat liver. J. Lipid Res. 18: 465-473.

11. Glickman, R. M., and K. Kirsch. 1974. The apoproteins of various size classes of human chylous fluid lipoproteins. Biochim. Biophys. Acta. 371: 255-266.

12. Schaefer, E. J., L. Jenkins, and H. B. Brewer, Jr. 1978. Human chylomicron apoprotein metabolism. Biochem. Biophys. Res. Commun. 80: 405-412.

13. Kostner, G., and A. Holasek. 1972. Characterization and quantitation of apoproteins from human chylomicrons. Biochemistry. 11: 1217-1223.

14. Rachmilewitz, D., J. J. Albers, D. R. Saunders, and M. Fainaru. 1978. Apoprotein synthesis by human duodenojejunal mucosa. Gastroenterology. 75: 667-682.

15. Glickman, R. M., P. H. R. Green, R. Lees, and A. Tall. 1978. Apoprotein A-I synthesis occurs in normal intestinal mucosa and in Tangier disease. N. Engl. J. Med. 299: 1424-1427.

16. Glickman, R. M., P. H. R. Green, R. Lees, S. E. Lux, and A. Kilgore. 1979. Immunofluorescence studies of apolipoprotein B in normal and abetalipoproteinemic intestinal mucosa. Gastroenterology. 76: 288-292.

17. Blomstrand, R., N. A. Thorn, and E. H. Ahrens, Jr. 1958. The absorption of fats, studied in a patient with chyluria. Am. J. Med. 24: 958-966.

18. Peterson, M. L. 1963. On the reesterification of fatty acids during absorption of fat: studies in patients with chyluria. Gastroenterology. 44: 779-786.

19. Hamilton, R. L., M. D. Williams, C. S. Fielding, and R. J. Havel. 1976. Discoidal bilayer structure of nascent high density lipoproteins from perfused rat liver. J. Clin. Invest. 58: 667-680.

20. Laurell, C. B. 1966. Quantitative estimation of proteins by electrophoresis in agarose gel containing antibodies. Anal. Biochem. 15: 45-52.
21. Albers, J. J., P. W. Wahl, V. G. Cabana, W. R. Hazzard, and J. J. Hoover. 1976. Quantitation of apolipoprotein $A_{1}$ of human high density lipoprotein. Metab. Clin. Exp. 25: 633-644.

22. Cheung, M. C., and J. J. Albers. 1977. The measurement of apolipoprotein A-I and A-II levels in men and women by immunoassay. J. Clin. Invest. 60: 43-50.

23. Folch, J., M. Lees, and G. H. Sloane Stanley. 1951. A simple method for the isolation and purification of total lipids from animal tissues. J. Biol. Chem. 226: 497-509.

24. Downing, D. T. 1968. Photodensitometry in the thinlayer chromatographic analysis of neutral lipids. $J$. Chromatogr. 38: 91-99.

25. Katz, S. S., G. G. Shipley, and D. M. Small. 1976. Physical chemistry of the lipids of human atherosclerotic lesions. J. Clin. Invest. 58: 200-211.

26. Lowry, O. M., N. R. Rosebrough, A. K. Farr, and R. J. Randall. 1951. Protein measurement with the Folin phenol reagent. J Biol. Chem. 193: 265-275.

27. Kessler, G., and M. Lederer. 1966. Fluorometric measurement of triglyceride. Automat. Anal. Chem. Technicon Symp. 341-344.

28. Imaizumi, K., M. Fainaru, and R. Havel. 1978. Composition of proteins of mesenteric lymph chylomicrons in the rat and alterations upon exposure of chylomicrons to blood serum and serum proteins. J. Lipid Res. 19: 712-722.

29. Swaney, J. B., M. Reese, and M. A. Eder. 1974. Polypeptide composition of rat high density lipoprotein: characterization by SDS gel electrophoresis. Biochem. Biophys. Res. Commun. 59: 513-519.

30. Marsh, J. B. 1976. Apoproteins of the lipoproteins in a non-recirculating perfusate of rat liver. J. Lipid Res. 17: 85-90.

31. Havel, R. J., and R. L. Hamilton. 1977. Synthesis and secretion of plasma lipoproteins and apolipoproteins. In Atherosclerosis IV. Proceedings of the Fourth International Symposium, Springer-Verlag, Berlin.

32. Blum, C. B., R. I. Levy, S. Eisenberg, M. Hall, III, R. H. Goebel, and M. Berman. 1977. High density lipoprotein metabolism in man. J. Clin. Invest. 60: 795-807.

33. Weisgraber, K. H., and R. W. Mahley. 1978. Apoprotein (E-A-II) complex of human plasma lipoproteins. I. Characterization of this mixed disulfide and its identification in a high density lipoprotein subfraction. J. Biol. Chem. 253: 6281-6288.

34. Shore, V. G., B. Shore, and S. B. Lewis. 1978. Isolation and characterization of two threonine-poor apolipoproteins of human plasma high density lipoproteins. Biochemistry. 17: 2174-2179.

35. Weisgraber, K. H., T. P. Bersot, and R. W. Mahley. 1978. Isolation and characterization of an apoprotein from the $d<1.006$ lipoproteins of human and canine lymph analogous to rat A-IV apoprotein. Biochem. Biophys. Res. Commun. 85: 287-292.

36. Tall, A., P. H. R. Green, and R. M. Glickman. 1978. Metabolic fate of chylomicrons, phospholipids and apoproteins in the rat. Circulation. 58: 11-15.

37. Redgrave, T. G., and D. M. Small. 1978. Transfer of surface components of chylomicrons to the high density lipoprotein fraction during chylomicron catabolism. Circulation. 58: 11-14.

38. Shepherd, J., C. J. Packard, and J. R. Patsch. 1978. Effects of dietary polyunsaturated and saturated fat on the properties of high density lipoproteins and the metabolism of apolipoprotein A-I. J. Clin. Invest. 6: 1582-1591. 